\title{
TWO WEIGHT NORM INEQUALITIES FOR FRACTIONAL ONE-SIDED MAXIMAL OPERATORS
}

\author{
F. J. MARTIN-REYES AND A. DE LA TORRE
}

(Communicated by J. Marshall Ash)

\begin{abstract}
In this paper we introduce a new maximal function, the dyadic one-sided maximal function. We prove that this maximal function is equivalent to the one-sided maximal function studied by the authors and Ortega in Weighted inequalities for one-sided maximal functions (Trans. Amer. Math. Soc. 319 (1990)) and by Sawyer in Weighted inequalities for the one-sided HardyLittlewood maximal functions (Trans. Amer. Math. Soc. 297 (1986)), but our function, being dyadic, is much easier to deal with, and it allows us to study fractional maximal operators. In this way we obtain a geometric proof of the characterization of the good weights for fractional maximal operators, answering a question raised by Andersen and Sawyer in Weighted norm inequalities for the Riemann-Liouville and Weyl fractional integral operators (Trans. Amer. Math. Soc. 308 (1988)). Our methods, avoiding complex interpolation, give also the case of different weights for the fractional maximal operator, which was an open problem.
\end{abstract}

\section{INTRODUCTION}

In [1] Andersen and Sawyer characterized the good weights for the fractional maximal function $M_{\alpha}^{+}$using complex interpolation and, as a consequence, were able to characterize the good weights for the fractional integral operators. Their methods seem to be restricted to the case of equal weights and raise the question of obtaining a geometric proof of the characterization of the good weights for $M_{\alpha}^{+}$.

We introduce a dyadic one-sided maximal function $M_{\alpha, D}^{+}$, and prove that it is pointwise equivalent to $M_{\alpha}^{+}$; furthermore, since our maximal function is dyadic, Sawyer's original technique [3] can be used to characterize the pairs of weights for which it is bounded (even in the case of different weights). We obtain a general condition and prove that in the case of equal weights it is equivalent to condition (1.5) in [1]. In this way we give a new proof of Theorem 1 in [1].

Throughout this paper $C$ will denote a positive constant, not necessarily the same at each occurrence. If $p>1$, its conjugate exponent will be denoted by $p^{\prime}$. For any measurable set $A$ and any positive function $g, \chi_{A}$ will denote the

Received by the editors February 8, 1990 and, in revised form, June 18, 1991.

1991 Mathematics Subject Classification. Primary 42B25.

Key words and phrases. One-sided fractional maximal functions, weighted inequalities.

Research supported by C.A.I.C.Y.T Grant (PB85-0434). 
characteristic function of $A,|A|$ its Lebesgue measure, and $g(A)$ the integral of $g$ over $A$. If $I=[a, b)$ is an interval, then we will denote by $I^{*}$ the interval $[b, 2 b-a)$.

\section{THE FRACTIONAL, ONE-SIDED, DYADIC MAXIMAL FUNCTION}

For each $x$ in $\mathbb{R}$, we consider the family of intervals $A_{x}=\{I=[a, b) ; I$ is dyadic and $0 \leq a-x<b-a\}$. Now for each locally integrable $f$ and $1>\alpha>0$, we define the one-sided, dyadic, fractional maximal function $M_{\alpha, D}^{+}$ as

$$
M_{\alpha, D}^{+} f(x)=\sup \left\{|I|^{\alpha-1} \int_{I}|f| ; I \in A_{x}\right\} .
$$

The interest of this maximal function lies in the fact that it is equivalent to the usual one-sided fractional maximal function

$$
M_{\alpha}^{+} f(x)=\sup _{\alpha>0} a^{\alpha-1} \int_{x}^{x+a}|f| .
$$

In order to be able to take averages away from $x$ it is convenient to introduce a new maximal function that is equivalent to $M_{\alpha}^{+}$,

$$
N_{\alpha}^{+} f(x)=\sup _{\alpha>0}\left(\frac{a}{2}\right)^{\alpha-1} \int_{x+a / 2}^{x+a}|f| .
$$

\section{(2.4) Proposition.}

$$
\begin{aligned}
M_{\alpha}^{+} f(x) & \leq\left(2^{1-\alpha}-1\right)^{-1} N_{\alpha}^{+} f(x), \\
N_{\alpha}^{+} f(x) & \leq 2^{1-\alpha} M_{\alpha}^{+} f(x) .
\end{aligned}
$$

Proof. It is enough to consider positive and bounded $f$ and take sups in the following obvious inequalities:

$$
\begin{gathered}
(a / 2)^{\alpha-1} \int_{x+a / 2}^{x+a} f \leq(a / 2)^{\alpha-1} \int_{x}^{x+a} f \leq 2^{1-\alpha} M_{\alpha}^{+} f(x), \\
a^{\alpha-1} \int_{x}^{x+a} f=a^{\alpha-1} \int_{x}^{x+a / 2} f+a^{\alpha-1} \int_{x+a / 2}^{x+a} f \leq 2^{\alpha-1} M_{\alpha}^{+} f(x)+2^{\alpha-1} N_{\alpha}^{+} f(x) .
\end{gathered}
$$

(2.5) Proposition. For each $\alpha$ there are two constants $P_{\alpha}$ and $Q_{\alpha}$ such that

$$
M_{\alpha}^{+} f(x) \leq P_{\alpha} M_{\alpha, D}^{+} f(x), \quad M_{\alpha, D}^{+} f(x) \leq Q_{\alpha} M_{\alpha}^{+} f(x) .
$$

Proof. Let us fix $f \geq 0$ and $x$ in $\mathbb{R}$. Let $I=[a, b) \in A_{x}$. Then

$$
|I|^{\alpha-1} \int_{I} f \leq(b-x)^{1-\alpha}|I|^{\alpha-1}(b-x)^{\alpha-1} \int_{x}^{b} f \leq 2^{1-\alpha} M_{\alpha}^{+} f(x) .
$$

Conversely, it is enough to consider the case in which $a$ is of the form $2^{k}$; let $I$ and $I^{*}$ be two dyadic intervals of length $2^{k-1}$, whose union covers $[x+a / 2, x+a)$. If $I \cup I^{*}$ is dyadic then $I \cup I^{*} \in A_{x}$ and

$$
\int_{x+a / 2}^{x+a} f \leq \int_{I \cup I^{*}} f \leq 2^{k(1-\alpha)} M_{\alpha, D}^{+} f(x) .
$$


If $I \cup I^{*}$ is not dyadic then let $I_{1}$ be the dyadic interval of length $2^{k}$ that contains $I^{*}$. It is clear that $I$ and $I_{1}$ belong to $A_{x}$, and then

$$
\int_{x+a / 2}^{x+a} f \leq \int_{I} f+\int_{I_{1}} f \leq\left(2^{(k-1)(1-\alpha)}+2^{k(1-\alpha)}\right) M_{\alpha, D}^{+} f(x) .
$$

In any case,

$$
\int_{x+a / 2}^{x+a} f \leq 2^{k(1-\alpha)}\left(1+2^{\alpha-1}\right) M_{\alpha, D}^{+} f(x)
$$

therefore, $N_{\alpha}^{+} f(x) \leq 2^{1-\alpha}\left(1+2^{\alpha-1}\right) M_{\alpha, D}^{+} f(x)$, and the result follows from (2.4).

(2.6) Theorem. For nonnegative functions $u, v$ and $1<p \leq q$, the following two conditions are equivalent.

(A) There exists $C$ such that for every nonnegative $f$

$$
\left(\int\left(M_{\alpha, D}^{+} f\right)^{q} u\right)^{1 / q} \leq C\left(\int f^{p} v\right)^{1 / p} .
$$

$\left(S_{p, q, \alpha, D}^{+}\right)$There exists $C$ such that for every dyadic interval $I=[a, b)$ such that $\int_{(-\infty, a)} u>0$, one has

$$
\int_{I \cup I^{*}} \sigma<\infty \text { and }\left(\int_{I \cup I^{*}}\left(M_{\alpha, D}^{+} \sigma \chi_{I^{*}}\right)^{q} u\right)^{1 / q} \leq C\left(\int_{I^{*}} \sigma\right)^{1 / p}
$$

where $\sigma=v^{1-p^{\prime}}$.

Proof. To prove the first part of (2.7) we observe that if for some dyadic interval $I=[a, b)$ is $\int_{I \cup I^{*}} \sigma=\infty$, while $\int_{(-\infty, a)} u>0$, then there is a function $f$ in $L_{p}(v)$ such that $\int_{I \cup I^{*}} f=\infty$. This implies that for points $x<a$, close to $a, M_{\alpha, D}^{+} f(x)=\infty$, contradicting $(A)$. For the second part just take $f=\sigma \chi_{I^{*}}$. The converse is a modification of Sawyer's argument [3]. Without loss of generality, we may assume that the length of our intervals is uniformly bounded. For each integer $k$, we consider the set $O_{k}=\left\{x ; M_{\alpha, D}^{+} f(x)>2^{k}\right\}$. Then for each $x$ in $O_{k}$ there is a dyadic interval $I_{x, k}$ such that $x \in I_{x, k}$ and $\int_{I_{x, k}^{*}} f>2^{k}\left|I_{x, k}^{*}\right|^{1-\alpha}$. From the definition of $M_{\alpha, D}^{+}$, it follows that $I_{x, k}$ is contained in $O_{k}$. Since our intervals are dyadic, we may choose for each $k$, a maximal, disjoint collection $I_{j, k}$ such that

(a) $\bigcup I_{j, k}=O_{k}$,

(b) $\int_{I_{j, k}^{*}} f>2^{k}\left|I_{j, k}^{*}\right|^{1-\alpha}$.

Now if we define $E_{j, k}=\left\{x \in I_{j, k} ; M_{\alpha, D}^{+} f(x) \leq 2^{k+1}\right\}$, we may write

$$
\begin{aligned}
\int\left(M_{\alpha, D}^{+} f\right)^{q} u & \leq 2^{q} \sum_{j, k} 2^{k q} u\left(E_{j, k}\right) \\
& \leq 2^{q} \sum_{j, k} u\left(E_{j, k}\right)\left(\left|I_{j, k}^{*}\right|^{\alpha-1} \int_{I_{j, k}^{*}} f\right)^{q} \\
& =2^{q} \sum_{j, k} \gamma_{j, k}\left(\sigma\left(I_{j, k}^{*}\right)^{-1} \int_{I_{j, k}^{*}} f \sigma^{-1} \sigma\right)^{q},
\end{aligned}
$$


where

$$
\gamma_{j, k}=u\left(E_{j, k}\right)\left(\left|I_{j, k}^{*}\right|^{\alpha-1} \int_{I_{j, k}^{*}} \sigma\right)^{q} .
$$

Following the argument in [3], it is enough to prove that the operator $T$, defined by $T g=\sigma\left(I_{j, k}^{*}\right)^{-1} \int_{I_{j, k}^{*}}|g| \sigma$ is of weak type $(1, q / p)$ with respect to the measures $\gamma_{j, k}$ in $\mathbb{Z} \times \mathbb{Z}$, and $\sigma d x$ in $\mathbb{R}$. We need then to prove that

$$
\sum\left\{\gamma_{j, k} ; T g(j, k)>\lambda\right\} \leq C\left(\lambda^{-1} \int|g| \sigma\right)^{q / p} \text {. }
$$

Since our intervals $I_{j, k}^{*}$ are dyadic, we may choose a maximal collection $I_{i}^{*}$ relative to the property $T g(j, k)>\lambda$. It is clear that for each $x$ in $E_{j, k}$,

$$
\left|I_{j, k}^{*}\right|^{\alpha-1} \int_{I_{j, k}^{*}} \sigma \leq M_{\alpha, D}^{+}\left(\sigma \chi_{I_{j, k}^{*}}\right)(x) .
$$

Therefore using (2.7) and the fact that $q / p>1$, we have

$$
\begin{aligned}
\sum\left\{\gamma_{j, k} ; T g(j, k)>\lambda\right\} & \leq \sum_{j, k} \int_{E_{j, k}} u\left(M_{\alpha, D}^{+}\left(\sigma \chi_{I_{j, k}^{*}}\right)(x)\right)^{q} \\
& \leq \sum_{i} \sum_{I_{j, k}^{*} \subseteq I_{i}^{*}} \int_{E j, k} u\left(M_{\alpha, D}^{+}\left(\sigma \chi_{I_{i}^{*}}\right)(x)\right)^{q} ;
\end{aligned}
$$

but $I_{j, k}^{*} \subset I_{i}^{*}$ implies $E_{j, k} \subset I_{i} \cup I_{i}^{*}$, and then

$$
\begin{aligned}
& \sum\left\{\gamma_{j, k} ; T g(j, k)>\lambda\right\} \leq \sum_{i} \int_{I_{i} \cup I_{i}^{*}} u\left(M_{\alpha, D}^{+}\left(\sigma \chi_{I_{i}^{*}}\right)(x)\right)^{q} \\
& \leq C \sum_{i}\left(\int_{I_{i}^{*}} \sigma\right)^{q / p} \leq C\left(\sum_{i} \int_{I_{i}^{*}} \sigma\right)^{q / p} \leq C\left(\lambda^{-1} \int|g| \sigma\right)^{q / p} .
\end{aligned}
$$

Remark. It follows from the proof that although (2.7) is stated only for dyadic intervals, it is equivalent to the same condition for any interval.

$\left(S_{p, q, \alpha, D}^{+}\right)$seems stronger than the usual $\left(S_{p}^{+}\right)$condition, but actually they are equivalent.

Proposition. The following two conditions are equivalent.

$\left(S_{p, q, \alpha, D}^{+}\right)$There exists $C$ such that for every interval $I$, with $\sigma\left(I \cup I^{*}\right)$ finite,

$$
\left(\int_{I \cup I^{*}}\left(M_{\alpha, D^{+}}^{+} \sigma \chi_{I^{*}}\right)^{q} u\right)^{1 / q} \leq C\left(\int_{I^{*}} \sigma\right)^{1 / p} .
$$

$\left(S_{p, q, \alpha}^{+}\right)$There exists $C$ such that for every interval $I$ with $\sigma(I)$ finite $\left(\int_{I}\left(M_{\alpha}^{+} \sigma \chi_{I}\right)^{q} u\right)^{1 / q} \leq C\left(\int_{I} \sigma\right)^{1 / p}$.

Proof. $\left(S_{p, q, \alpha, D}^{+}\right)$implies $\left(S_{p, q, \alpha}^{+}\right)$follows immediately from the equivalence of $M_{\alpha}^{+}$and $M_{\alpha, D}^{+}$. Conversely, let $I$ be any interval. Then

$$
\begin{aligned}
\left(\int_{I \cup I^{*}}\left(M_{\alpha, D^{*}}^{+} \sigma \chi_{I^{*}}\right)^{q} u\right)^{1 / q} & \leq C\left(\int_{I}\left(M_{\alpha}^{+} \sigma \chi_{I^{*}}\right)^{q} u\right)^{1 / q}+C\left(\int_{I^{*}}\left(M_{\alpha}^{+} \sigma \chi_{I^{*}}\right)^{q} u\right)^{1 / q} \\
& \leq C\left(\int_{I}\left(M_{\alpha}^{+} \sigma \chi_{I^{*}}\right)^{q} u\right)^{1 / q}+C\left(\int_{I^{*}} \sigma\right)^{1 / p} .
\end{aligned}
$$


Therefore, it is enough to show that for every $I$ there is a $C$ such that

$$
\left(\int_{I}\left(M_{\alpha}^{+} \sigma \chi_{I^{*}}\right)^{q} u\right)^{1 / q} \leq C\left(\int_{I^{*}} \sigma\right)^{1 / p} .
$$

Let $I=[a, b) ; I^{*}=[b, c)$. If $\int_{I} \sigma \leq \int_{I^{*}} \sigma$ then

$$
\begin{aligned}
\left(\int_{I}\left(M_{\alpha}^{+} \sigma \chi_{I^{*}}\right)^{q} u\right)^{1 / q} & \leq\left(\int_{I \cup I^{*}}\left(M_{\alpha}^{+} \sigma \chi_{I^{*}}\right)^{q} u\right)^{1 / q} \\
& \leq C\left(\int_{I \cup I^{*}} \sigma\right)^{1 / p} \leq 2^{1 / p} C\left(\int_{I^{*}} \sigma\right)^{1 / p} .
\end{aligned}
$$

If $\int_{I} \sigma \geq \int_{I^{*}} \sigma$, then we choose a sequence $x_{0}=b>x_{1}>x_{2}>\cdots>x_{k}>$ $, \ldots, x_{N-1}>x_{N}=a$ such that for $k=0,1, \ldots, N-1, \int_{x_{k}}^{c} \sigma=2^{k} \int_{b}^{c} \sigma$ and $\int_{a}^{c} \sigma=r \int_{b}^{c} \sigma, 2^{N-1}<r \leq 2^{N}$. It follows that $\int_{x_{k}}^{x_{k-1}} \sigma=2^{k-1} \int_{b}^{c} \sigma, 0<k<N$, while $\int_{a}^{x_{N-1}} \sigma \leq 2^{N-1} \int_{b}^{c} \sigma$. Now if $x_{k}<x<x_{k-1}, 1<k \leq N$, and $y \in I^{*}$, then

$$
\int_{x}^{y} \sigma \chi_{(b, c)}=\int_{b}^{y} \sigma \leq \int_{b}^{c} \sigma=2^{-(k-2)} \int_{x_{k-1}}^{x_{k-2}} \sigma \leq 2^{-(k-2)} \int_{x}^{y} \sigma .
$$

Multiplying both sides of this inequality by $(y-x)^{\alpha-1}$ and taking the sup, we get that for any $x, x_{k}<x<x_{k-1}$, one has

$$
M^{+}\left(\sigma \chi_{(b, c)}\right)(x) \leq 2^{-(k-2)} M^{+}\left(\sigma \chi_{(x, c)}\right)(x), \quad k=2, \ldots, N,
$$

while for $k=1$ we have the trivial estimate

$$
M^{+}\left(\sigma \chi_{(b, c)}\right)(x) \leq M^{+}\left(\sigma \chi_{(x, c)}\right)(x), \quad x_{1}<x<b .
$$

Therefore

$$
\begin{aligned}
\int_{a}^{b}( & \left.M^{+}\left(\sigma \chi_{(b, c)}\right)\right)^{q} u=\sum_{1}^{N} \int_{x_{k}}^{x_{k-1}}\left(M^{+}\left(\sigma \chi_{(b, c)}\right)\right)^{q} u \\
\quad \leq & \sum_{2}^{N} 2^{-(k-2) q} \int_{x_{k}}^{x_{k-1}}\left(M^{+}\left(\sigma \chi_{\left(x_{k}, c\right)}\right)\right)^{q} u+\int_{x_{1}}^{b}\left(M^{+}\left(\sigma \chi_{\left(x_{1}, c\right)}\right)\right)^{q} u \\
& \leq \sum_{2}^{N} 2^{-(k-2) q} \int_{x_{k}}^{c}\left(M^{+}\left(\sigma \chi_{\left(x_{k}, c\right)}\right)\right)^{q} u+\int_{x_{1}}^{c}\left(M^{+}\left(\sigma \chi_{\left(x_{1}, c\right)}\right)\right)^{q} u \\
& \leq \sum_{2}^{N} 2^{-(k-2) q}\left(\int_{x_{k}}^{c} \sigma\right)^{q / p}+\left(\int_{x_{1}}^{c} \sigma\right)^{q / p} \\
& \leq\left(\sum_{2}^{N} 2^{-(k-2) q} 2^{k q / p}+2^{q / p}\right)\left(\int_{b}^{c} \sigma\right)^{q / p} \leq C\left(\int_{b}^{c} \sigma\right)^{q / p} .
\end{aligned}
$$

\section{THE CASE OF EQUAL WEIGHTS}

In [1] it is proved that if $1<p \leq q, q^{-1}=p^{-1}-\alpha$, a necessary and sufficient condition for $M_{\alpha}^{+}$to be a bounded operator from $L_{p}\left(u^{p} d x\right)$ to $L_{q}\left(u^{q} d x\right)$ is 
$\left(A_{p, q}^{+}\right)$There exists $C$ such that for any $a$ and any positive $h$

$$
\left(h^{-1} \int_{(a-h, a)} u^{q}\right)^{1 / q}\left(h^{-1} \int_{(a, a+h)} u^{-p^{\prime}}\right)^{1 / p^{\prime}} \leq C .
$$

We will give a direct proof of the equivalence of this condition with condition $\left(S_{p, q, \alpha, D}^{+}\right)$, thus obtaining a geometric proof of this result. Observe that $\left(u^{q}, u^{p}\right)$ satisfies $\left(S_{p, q, \alpha, D}^{+}\right)$if

$$
\left(\int_{(a-h, a+h)}\left(M_{\alpha}^{+} u^{-p^{\prime}} \chi_{(a, a+h)}\right)^{q} u^{q}\right)^{1 / q} \leq C\left(\int_{(a, a+h)} u^{-p^{\prime}}\right)^{1 / p}
$$

which of course implies

$$
\left(\int_{(a-h, a)}\left(M_{\alpha}^{+} u^{-p^{\prime}} \chi_{(a, a+h)}\right)^{q} u^{q}\right)^{1 / q} \leq C\left(\int_{(a, a+h)} u^{-p^{\prime}}\right)^{1 / p},
$$

but for any $x$ in $(a-h, a), h^{\alpha-1} \int_{(a, a+h)} u^{-p^{\prime}} \leq M_{\alpha}^{+} u^{-p^{\prime}} \chi_{(a, a+h)}(x)$ and $\left(A_{p, q}^{+}\right)$ follows.

To prove the converse we will use the equivalence between $\left(S_{p, q, \alpha, D}^{+}\right)$and $\left(S_{p, q}^{+}\right)$and prove that $\left(A_{p, q}^{+}\right)$implies $\left(S_{p, q, \alpha}^{+}\right)$using the method of [2]. Let $I$ be fixed. A similar argument to the one used in Proposition (2.4) proves that for every $x$ there exists an $h$ (that depends on $x$ ) and a constant $C_{\alpha}$ (that depends only on $\alpha$ ) such that

$$
M_{\alpha}^{+}\left(\chi_{I} u^{-p^{\prime}}\right)(x) \leq C_{\alpha} h^{\alpha-1} \int_{(x+h / 2, x+h)} u^{-p^{\prime}}
$$

Using $\left(A_{p, q}^{+}\right)$and the relationship between $p, q$, and $\alpha$, we obtain

$$
\begin{aligned}
\left(M_{\alpha}^{+}\left(\chi_{I} u^{-p^{\prime}}\right)(x)\right)^{q} & \leq C_{\alpha} h^{\alpha q+p^{\prime}}\left(\int_{(x, x+h / 2)} u^{q}\right)^{-p^{\prime}} \\
& =C_{\alpha}\left(h\left(\int_{(x, x+h / 2)} u^{q}\right)^{-p^{\prime} /\left(\alpha q+p^{\prime}\right)}\right)^{\alpha q+p^{\prime}}
\end{aligned}
$$

Let us now define $s=\alpha q+p^{\prime}$ and $\beta=1-p^{\prime} /\left(\alpha q+p^{\prime}\right)$, and let us consider the operator $M_{u^{q}, \beta} f(x)=\sup _{x \in I} u^{q}(I)^{\beta-1} \int_{I}|f| u^{q}$. Our last inequality can now be written as

$$
\left(M_{\alpha}^{+}\left(\chi_{I} u^{-p^{\prime}}\right)(x)\right)^{q} \leq C_{\alpha}\left(M_{u^{q}, \beta}\left(u^{-q} \chi_{I}\right)\right)^{2} .
$$

But it is well known [3] that $M_{u^{q}, \beta}$ maps $L_{t}\left(u^{q}\right)$ into $L_{s}\left(u^{q}\right)$, provided $s^{-1}=$ $t^{-1}-\beta$; i.e., $t=s /(1+s \beta)$. Integrating both sides of (3.1) over $I$ and using this result, one gets

$$
\begin{aligned}
\left(\int_{I}\left(M_{\alpha}^{+}\left(\chi_{I} u^{-p^{\prime}}\right)(x)\right)^{q} u^{q}\right)^{1 / q} & \leq C_{\alpha}\left(\int_{I}\left(M_{u^{q}, \beta}\left(u^{-q} \chi_{I}\right)\right)^{s} u^{q}\right)^{1 / q} \\
& \leq C_{\alpha}\left(\int_{I} u^{-q t} u^{q}\right)^{s / q t}
\end{aligned}
$$


But is easy to check that $t=p s / q$ and $q-q t=-p^{\prime}$, and therefore, we have proved

$$
\left(\int_{I}\left(M_{\alpha}^{+}\left(\chi_{I} u^{-p^{\prime}}\right)(x)\right)^{q} u^{q}\right)^{1 / q} \leq C_{\alpha}\left(\int_{I} u^{-p^{\prime}}\right)^{1 / p}
$$

which is $\left(S_{p, q, \alpha}^{+}\right)$.

\section{REFERENCES}

1. K. F. Andersen and E. T. Sawyer, Weighted norm inequalities for the Riemann-Liouville and Weyl fractional integral operators, Trans. Amer. Math. Soc. 308 (1988), 547-557.

2. F. J. Martin-Reyes, P. Ortega, and A. de la Torre, Weighted inequalities for one-sided maximal functions, Trans. Amer. Math. Soc. 319 (1990), 517-534.

3. E. T. Sawyer, $A$ characterization of a two-weighted norm inequality for maximal operators, Studia Math. 75 (1982), 1-11.

4. _ Weighted inequalities for the one-sided Hardy-Littlewood maximal functions, Trans. Amer. Math. Soc. 297 (1986), 53-61.

Departamento de Análisis Matemático, Facultad de Ciencias, Universidad de Málaga, 29071 Málaga, Spain

E-mail address, F. J. Martin-Reyes: MARTIN_REYES@CCUMA.UMA.ES

E-mail address, A. de la Torre: TORRE_R@CCUMA.UMA.ES 